Claremont Colleges

Scholarship@ Claremont

All HMC Faculty Publications and Research

HMC Faculty Scholarship

$1-1-2006$

\title{
The Motion of a Thin Liquid Film Driven by Surfactant and Gravity
}

Michael Shearer

North Carolina State University at Raleigh

Rachel Levy

Harvey Mudd College

\section{Recommended Citation}

Shearer, M., and R. Levy. "The Motion of a Thin Film Driven by Surfactant and Gravity." SIAM Journal on Applied Mathematics 66.5 (2006): 1588-1609. DOI: $10.1137 / 050637030$

This Article is brought to you for free and open access by the HMC Faculty Scholarship at Scholarship @ Claremont. It has been accepted for inclusion in All HMC Faculty Publications and Research by an authorized administrator of Scholarship @ Claremont. For more information, please contact scholarship@cuc.claremont.edu. 


\title{
THE MOTION OF A THIN LIQUID FILM DRIVEN BY SURFACTANT AND GRAVITY*
}

\author{
R. LEVY $^{\dagger}$ AND M. SHEARER ${ }^{\ddagger}$
}

\begin{abstract}
We investigate wave solutions of a lubrication model for surfactant-driven flow of a thin liquid film down an inclined plane. We model the flow in one space dimension with a system of nonlinear PDEs of mixed hyperbolic-parabolic type in which the effects of capillarity and surface diffusion are neglected. Numerical solutions reveal distinct patterns of waves that are described analytically by combinations of traveling waves, some with jumps in height and surfactant concentration gradient. The various waves and combinations are strikingly different from what is observed in the case of flow on a horizontal plane. Jump conditions admit new shock waves sustained by a linear surfactant wave traveling upstream. The stability of these waves is investigated analytically and numerically. For initial value problems, a critical ratio of upstream to downstream height separates two distinct long-time wave patterns. Below the critical ratio, there is also an exact solution in which the height is piecewise constant and the surfactant concentration is piecewise linear and has compact support.
\end{abstract}

Key words. PDE, surfactants, hyperbolic-parabolic system

AMS subject classifications. 35G30, 35M10, 76Z05, 76D08

DOI. $10.1137 / 050637030$

1. Introduction. Spatial variations in surface tension on the free surface of a fluid induce a surface force, known as a Marangoni force [8]. Such variations can occur through temperature changes $[2,4]$ or by introducing surfactants. In thin film flow, surfactants have been studied in the context of industrial coating processes $[9,16]$ and as a component in the treatment of premature babies, whose lungs are in danger of collapse due to insufficient natural surfactant [3, 11]. Both applications have motivated extensive recent research on thin films driven by surfactant $[12,13,17,18]$.

In much of the research into surfactant spreading, the effect of gravity in driving the flow has been assumed to be negligible [10]. In this paper, we consider flow on an inclined plane, building on the work of Edmonstone, Craster, and Matar $[6,7]$ that explores the effect of adding gravity to the driving force. Our results demonstrate that gravity has a profound effect and probably should not be neglected in simulations of surfactant spreading.

For constant Marangoni force, as in studies of thermally driven thin films [2, $4,8]$, the equations of motion are reasonably represented by a scalar fourth order PDE, known as the thin film equation. In the presence of surfactant, however, the Marangoni force is not constant; the density and motion of the surfactant molecules are modeled by an additional equation. The full model, derived using lubrication theory in [3], consists of a system of two nonlinear coupled PDEs for the film height and the surfactant concentration.

The PDE system exhibits a complicated combination of wave-like structures in the solution of initial value and boundary value problems $[6,7]$. The complexity comes

${ }^{*}$ Received by the editors July 28, 2005; accepted for publication (in revised form) March 28, 2006; published electronically June 9, 2006. This work was supported by NSF grant DMS-0244491.

http://www.siam.org/journals/siap/66-5/63703.html

$\dagger$ Department of Mathematics, Duke University, Durham, NC 27708 (rachel.levy@gmail.com).

${ }^{\ddagger}$ Department of Mathematics and Center for Research in Scientific Computation, North Carolina State University, Raleigh, NC 27695, and Department of Mathematics, Duke University, Durham, NC 27708 (shearer@math.ncsu.edu). 
about in part because the underlying equation for the transport of surfactant is a degenerate parabolic equation resembling the porous medium equation, the degeneracy occurring at zero surfactant concentration. In contrast with the scalar thin film equation [15], we cannot appeal to the theory of hyperbolic equations to predict the long-time behavior of solutions, and indeed we find that it is different from the combination of shocks and rarefaction waves of the hyperbolic theory. Nonetheless, we explore the point of view that long-time behavior of the underlying equations should be predictable from basic information in the initial and boundary conditions, and it should be given by combinations of similarity solutions of the equations.

The underlying equations we study are derived from the full lubrication system (in section 2) by omitting terms related to surface diffusion and capillarity, i.e., taking the Péclet number to be infinite and the capillary number to be zero. We also neglect a second order diffusive term that is small for steep inclines. This enables us to focus on the underlying structure of solutions by analogy with the connection between hyperbolic systems and their parabolic regularization.

Numerical simulations of the reduced system, described in section 3, contain discontinuities in film height and surfactant concentration gradient. These solutions have a recognizable structure, and the goal of much of the rest of the paper is to explain the structure analytically. There is extensive numerical evidence [6] that the omitted regularizing terms primarily smooth the discontinuities without changing their structure or speeds, provided the coefficients are small.

The analysis of traveling waves in section 4 and jump conditions in section 5 reveals a surprisingly rich variety of individual waves. In section 6 , we show how these waves can be combined to explain the wave patterns in the numerical simulations. However, the combination is possible only below a critical value of the ratio $h_{R} / h_{L}$ of downstream height $h_{R}$ to upstream height $h_{L}$. This analysis is used in section 7 to generate special exact solutions that are piecewise constant in $h$ and piecewise linear in $\Gamma$, with $\Gamma$ having compact support.

Above the critical value a different wave pattern emerges, described through numerical experiments in section 7. A notable feature of these new patterns is a hyperbolic precursor wave propagating ahead of the surfactant front. Also in this section we probe the critical value in PDE simulations and investigate the stability of individual waves predicted by jump conditions. In section 8 we summarize the catalogue of new individual waves and discuss the results in the context of ongoing research into the role of gravity in surfactant spreading.

2. The model. Consider a flat solid substrate, inclined as shown in Figure 1, in which $z=\tilde{h}(x, y, t)$ is the height of a thin film flowing down the slope. On the surface of the film is a layer of surfactant with concentration $\tilde{\Gamma}(x, y, t)$, which measures the density of surfactant molecules on the free surface. The surfactant is assumed to be immiscible and does not add to the height of the film. ${ }^{1}$

The full multidimensional model consists of a system of two nonlinear PDEs derived from the Navier-Stokes equations and the well-known lubrication approximation $[1,6,13,19]$ :

$$
h_{t}+\nabla \cdot\left[\mathbf{C} \frac{h^{3}}{3} \nabla \nabla^{2} h-\mathbf{G} \cos \theta \frac{h^{3}}{3} \nabla h-\frac{h^{2}}{2} \nabla \Gamma\right]+\left[\mathbf{G} \sin \theta \frac{h^{3}}{3}\right]_{x}=0,
$$

\footnotetext{
${ }^{1}$ The amount of surfactant is also assumed to be below the critical micelle concentration [5].
} 


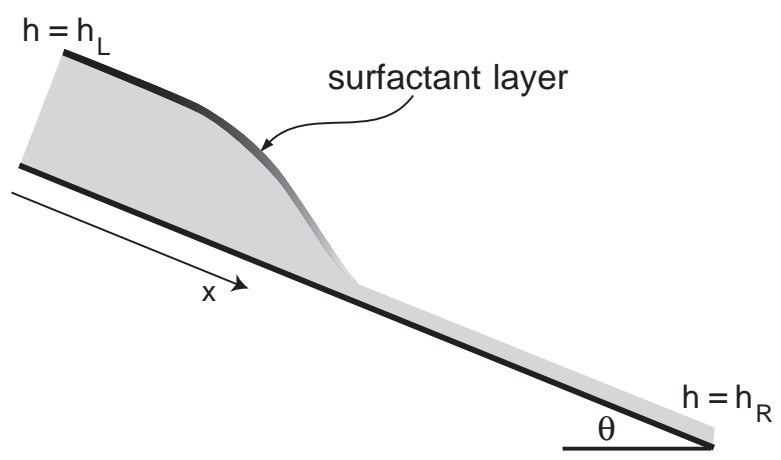

FIG. 1. A thin film on an inclined substrate with partial coating by surfactant of varying concentration and negligible height.

$$
\Gamma_{t}+\nabla \cdot\left[\mathbf{C} \frac{h^{2}}{2} \Gamma \nabla \nabla^{2} h-\mathbf{G} \cos \theta \frac{h^{2}}{2} \Gamma \nabla h-h \Gamma \nabla \Gamma\right]+\left[\mathbf{G} \sin \theta \frac{h^{2}}{2} \Gamma\right]_{x}-\mathbf{D} \nabla^{2} \Gamma=0 .
$$

Here $h, \Gamma$ are dimensionless variables: $h=\tilde{h} / H, \Gamma=\tilde{\Gamma} / \Gamma_{m}$, where $H$ is a characteristic length scale for the film thickness, and $\Gamma_{m}$ is a typical surfactant concentration. The system contains nondimensional parameters associated with gravity, $\mathbf{G}=\frac{\rho g H L}{\Pi}$, surface diffusion $\mathbf{D}=\frac{1}{\mathbf{P e}}=\frac{\mu D_{s}}{\Pi H}$ (where $\mathbf{P e}$ is the Péclet number), and capillarity $\mathbf{C}=\frac{\epsilon^{2} \sigma_{m}}{\Pi}$. These parameters depend on density $\rho$, gravity $g$, viscosity $\mu$, and surface diffusivity $D_{s}$ of the surfactant, all taken to be constant. They also depend on a characteristic length $L$ of the film and on the small parameter $\epsilon=\frac{H}{L}$. The spreading pressure $\Pi$ is given by $\Pi=\sigma_{0}-\sigma_{m}$, where $\sigma_{0}$ is the surface tension in the absence of surfactant, and $\sigma_{m}$ is a typical reduced surface tension in the presence of a typical concentration of surfactant. The spreading pressure $\Pi$ is related to the Marangoni number, which after nondimensionalization is effectively set to 1 . Note that in (2.1), (2.2) we have used a linear relation $\sigma=1-\Gamma$ (in nondimensional form) between surface tension and surfactant concentration. We refer the reader to [6] for typical values of the parameters.

In this paper, we consider a reduced model in which the variables are considered to be independent of the transverse variable $y$, and the regularizing effects of capillarity and surface diffusion are neglected by taking $\mathbf{C}=0$ and $\mathbf{D}=0$. Letting $\alpha=\mathbf{G} \sin \theta$, the reduced equations are

$$
\begin{aligned}
h_{t}-\frac{1}{2}\left(h^{2} \Gamma_{x}\right)_{x}+\frac{\alpha}{3}\left(h^{3}\right)_{x} & =0, \\
\Gamma_{t}-\left(h \Gamma \Gamma_{x}\right)_{x}+\frac{\alpha}{2}\left(h^{2} \Gamma\right)_{x} & =0 .
\end{aligned}
$$

In this system we have also neglected the gravity terms with coefficient $\mathbf{G} \cos \theta$. This coefficient is small for $\theta$ near $\frac{\pi}{2}$, where it has a minor smoothing effect on solutions [6]. It is well known that fourth order diffusion gives rise to a capillary ridge [20], which is not captured in the reduced system. Neither capillarity nor surface diffusion affects wave speeds significantly, at least for small values of $\mathbf{C}$ and $\mathbf{D}$, as verified numerically in [6]. 
It is perhaps helpful to compare this system to familiar PDEs. For a given function $\Gamma(x, t),(2.3)$ would be a scalar conservation law

$$
h_{t}+f(h)_{x}=0
$$

with $f(h)=-\frac{1}{2} h^{2} \Gamma_{x}+\frac{\alpha}{3} h^{3}$. Similarly, for a given $h(x, t),(2.4)$ resembles a porous medium equation. Specifically, if $h$ is constant, and $\alpha=0$, then (2.4) is

$$
\Gamma_{t}=\frac{1}{2} h\left(\Gamma^{2}\right)_{x x}
$$

Consequently, we may expect discontinuities in $h$ and $\Gamma_{x}$, while $\Gamma$ itself remains continuous; this would be typical in a quasi-linear conservation law for $h$ and a porous medium equation for $\Gamma$.

As this discussion suggests, the system of equations is of parabolic-hyperbolic type. In a series of papers [17, 18], Renardy examined analytical issues such as local existence, propagation speed, and formation of shocks in the case $\alpha=0$. While some of these results may generalize to the case $\alpha>0$, we do not pursue this line of analysis in this paper.

3. Numerical experiments I. The numerical algorithm used to compute solutions of the system of PDEs (2.3), (2.4) is a first order composite finite difference scheme that couples a fully implicit time step and central spatial differences for second order derivatives with an upwind scheme for the more hyperbolic first order terms (with coefficient $\alpha$ in (2.3), (2.4)).

We define a spatial finite difference operator acting on $u_{j}^{n}=u\left(x_{j}, t_{n}\right)$ as

$$
\left(\delta_{x} u\right)_{j+\frac{1}{2}}^{n} \equiv \frac{u_{j+1}^{n}-u_{j}^{n}}{\Delta x} .
$$

We also use standard notation for spatial averages:

$$
(\bar{u})_{j+\frac{1}{2}}^{n} \equiv \frac{u_{j+1}^{n}+u_{j}^{n}}{2},
$$

and let $\lambda \equiv \frac{\Delta t}{\Delta x}$. The finite difference scheme is then defined as

$$
\begin{aligned}
& h_{j}^{n+1}=h_{j}^{n}+\lambda\left(\frac{1}{2}\left(\bar{h}^{2} \delta_{x} \Gamma\right)_{j+\frac{1}{2}}^{n+1}-\frac{1}{2}\left(\bar{h}^{2} \delta_{x} \Gamma\right)_{j-\frac{1}{2}}^{n+1}-\frac{\alpha}{3}\left(\left(h^{3}\right)_{j}^{n}-\left(h^{3}\right)_{j-1}^{n}\right)\right), \\
& \Gamma_{j}^{n+1}=\Gamma_{j}^{n}+\lambda\left(\left(\bar{h} \bar{\Gamma} \delta_{x} \Gamma\right)_{j+\frac{1}{2}}^{n+1}-\left(\bar{h} \bar{\Gamma} \delta_{x} \Gamma\right)_{j-\frac{1}{2}}^{n+1}-\frac{\alpha}{2}\left(\left(h^{2} \Gamma\right)_{j}^{n}-\left(h^{2} \Gamma\right)_{j-1}^{n}\right)\right) .
\end{aligned}
$$

The resulting nonlinear system is solved with Newton's method; the composite scheme is formally first order in both time and space.

The goal of our analysis is to understand the structure of long-time solutions as a function only of boundary data. In order to simulate initial value problems with initial data in which $h$ and $\Gamma$ are constant outside an interval, and $\Gamma=0$ downstream, we impose simple boundary conditions at the end points of the computational domain $\left[0, x_{\max }\right]$ : 

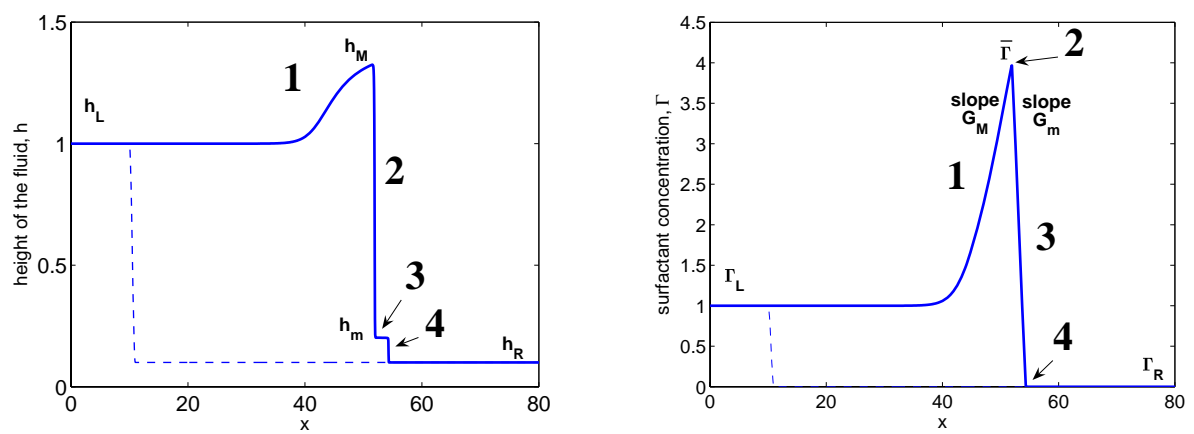

FIG. 2. Typical solution profiles $h(x, 60)$ (left) and $\Gamma(x, 60)$ (right) with $h_{R}=0.1$. Initial data $h(x, 0)$ and $\Gamma(x, 0)$ are represented by dashed lines. Numbered labels: (1) nonlinear traveling wave; (2) jump in $h, \Gamma_{x}$; (3) linear traveling wave with $\Gamma$ linear and $h$ constant; (4) jump in $h, \Gamma_{x}$.

$$
h(0, t)=h_{L}, \quad \Gamma(0, t)=\Gamma_{L} ; \quad h\left(x_{\max }, t\right)=h_{R}, \quad \Gamma\left(x_{\max }, t\right)=0 .
$$

In the PDE simulations, we exploit the following scalings in the equations. Let $h=H(x, t), \Gamma=g(x, t)$ satisfy $(2.3),(2.4)$ with $H(0, t)=1, g(0, t)=1$, and $\alpha=1$. Then $h, \Gamma$ given by

$$
h(x, t)=h_{L} H\left(\frac{\alpha h_{L}}{\Gamma_{L}} x, \frac{\alpha^{2} h_{L}^{3}}{\Gamma_{L}} t\right) \quad \text { and } \quad \Gamma=\Gamma_{L} g\left(\frac{\alpha h_{L}}{\Gamma_{L}} x, \frac{\alpha^{2} h_{L}^{3}}{\Gamma_{L}} t\right)
$$

satisfy (2.3), (2.4) with $h(0, t)=h_{L}, \Gamma(0, t)=\Gamma_{L}$, provided $\Gamma_{L}>0$. Thus, to explore variation in wave structures, we can fix $\alpha=h_{L}=\Gamma_{L}=1$ and vary $h_{R}$ (note that $\left.\Gamma_{R}=0\right)$. We employ this simplification in numerical simulations. In the analysis, we retain all the parameters so that their effect can be seen explicitly.

Figure 2 contains graphs of numerical simulations of (2.3), (2.4) at time $t=60$. The initial data (indicated by dashed lines) contain a single (smoothed) jump in $h$ from $h_{L}=1.0$ to $h_{R}=0.1$ and in $\Gamma$ from $\Gamma_{L}=1.0$ to $\Gamma_{R}=0$. The same structure emerges from the same upstream and downstream heights for more general smooth height data (including oscillatory data) after transients have died away. The figure is annotated to show the broad wave structure of the solution. In the graph of $h$, note that at (1) the height increases monotonically from the fixed boundary value $h_{L}$ to a maximum height $h_{M}$; at (2) there is a jump from $h_{M}$ to the height $h_{m}$ of the horizontal "step" at (3). Finally, the step contains a second jump at (4) from $h_{m}$ to the precursor layer of fixed height $h_{R}$.

The graph of the surfactant concentration $\Gamma$ has jumps at the same locations as those of the height, but the jumps are in the slope $\Gamma_{x}$ (henceforth denoted by $G$, as in the figure), while $\Gamma$ is continuous. The surfactant concentration increases monotonically (1) to a maximum concentration $\Gamma_{0}$ at the corner (2), where there is a jump in $\Gamma_{x}$ from $G_{M}>0$ to $G_{m}<0$. In (3), $\Gamma$ appears to be linear, extending down to $\Gamma=0$, where there is a second jump (4) in $\Gamma_{x}$ from $G_{m}<0$ to zero.

As the spatial grid is refined, the discontinuities are better resolved, and no additional data points appear in the discontinuities. We demonstrate this for the jump in height in Figure 3, in which the number of grid points is doubled from 2500 $(\Delta x=0.012)$ to $5000(\Delta x=0.006)$. 

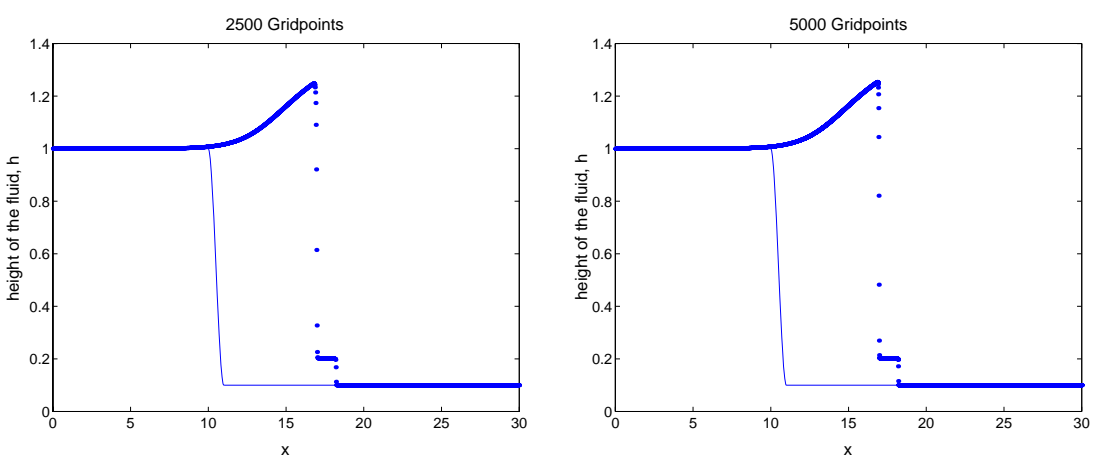

FIG. 3. Grid refinement to distinguish the discontinuity in height from a steep gradient. The coarse grid on the left has 2500 points; the fine grid on the right has 5000 points. The number of points in the shock is approximately the same in both cases.
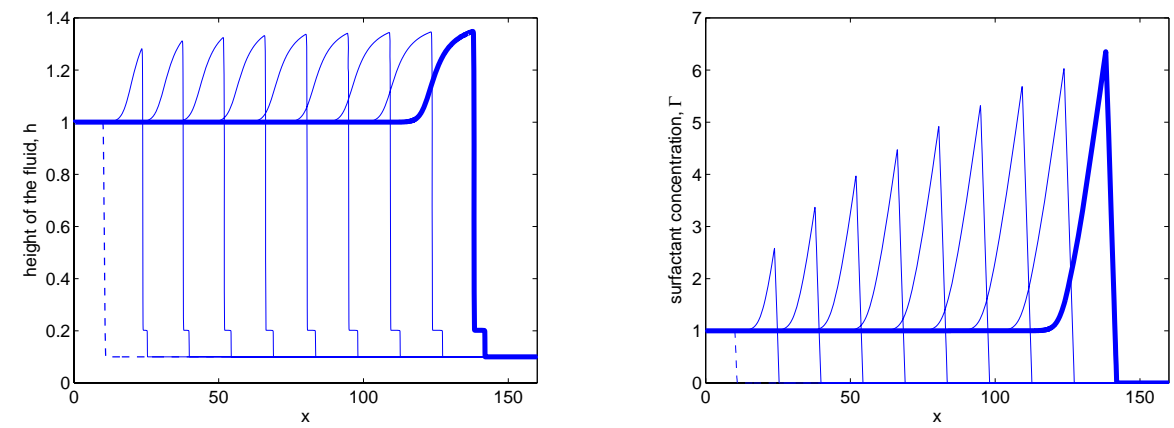

FIG. 4. Numerical solutions showing the evolution of $h$ and $\Gamma$ for $h_{R}=0.1$. The profiles are separated by 40 time steps, and the final profiles at $t=180$ are in bold. The computational time step is $\Delta t=0.001$, and the grid is fairly coarse with $\Delta x=0.04$.

Figure 4 illustrates the evolution of the solution, with a dashed line indicating the initial profile. After a rapid initial transient between the first two plots, the maximum height $h$ slowly asymptotes to a constant value, whereas the maximum surfactant concentration $\Gamma$ increases without bound. (Note that surfactant is supplied from the left boundary.) The step height $h_{m}$ develops at a very early time and remains constant, although the width of the step increases slowly.

To visualize the data from Figure 4 in a different way, in Figure 5 we plot the curves $(h(x, t), \Gamma(x, t)), 0<x<x_{\max }$, for various values of $t>0$. Starting from $\left(h_{L}, \Gamma_{L}\right)=(1,1)$, there is a sequence of curves on the right that appears to be converging. A jump in $h$ occurs at the maximum value of $\Gamma$, which is increasing in time. On the left of the figure, $\Gamma$ decreases to zero at a height $h$ which is constant in space and time. The final step occurs on the $h$-axis.

4. Traveling waves. In this section we explore analytical solutions of the PDE (2.3), (2.4) that capture some of the features observed in the numerical simulations. The traveling waves we consider are smooth solutions $h=\hat{h}(x-c t), \Gamma=\hat{\Gamma}(x-c t)$ that move with constant speed $c$. Substituting into system (2.3), (2.4) and integrating 


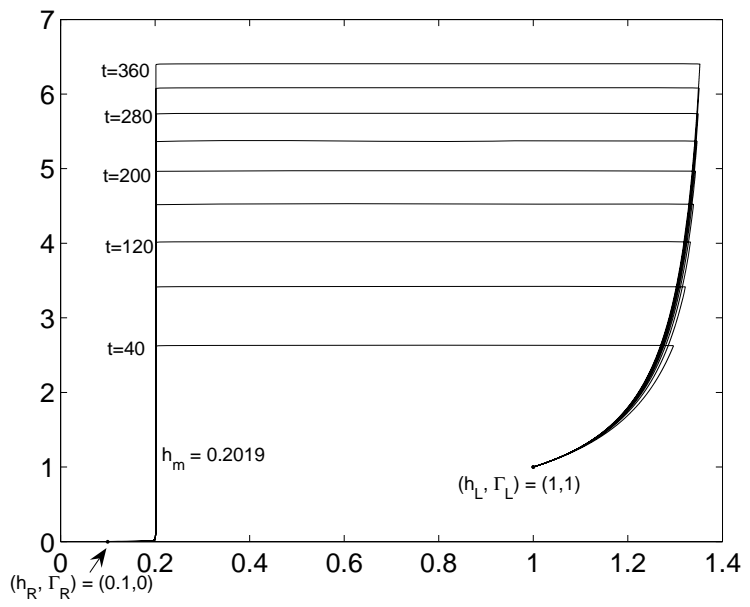

FIG. 5. Phase portrait of numerical PDE solutions $(h, \Gamma)$ with $h_{R}=0.1$.

once, we obtain the ODE system (dropping the hats)

$$
\begin{aligned}
& -c h-\frac{1}{2} h^{2} \Gamma^{\prime}+\frac{\alpha}{3} h^{3}=K_{1}, \\
& -c \Gamma-h \Gamma \Gamma^{\prime}+\frac{\alpha}{2} h^{2} \Gamma=K_{2}
\end{aligned}
$$

in which $\Gamma^{\prime}=\frac{d \Gamma}{d \xi}, \xi=x-c t$. First, we observe that there are traveling waves in which $h$ is constant and $\Gamma$ is linear. We refer to these traveling waves as simple traveling waves. Note that simple traveling waves should be considered to be defined only for values of $x-c t$ where $\Gamma$ is nonnegative.

TheOREM 4.1. Let $h>0$ be constant, and let $\Gamma=\Gamma_{0}+G \xi$, with $G=\Gamma^{\prime}=$ constant. Then (4.1) is satisfied identically with $K_{1}=-c h-\frac{1}{2} h^{2} G+\frac{\alpha}{3} h^{3}$, and (4.2) is satisfied if and only if $K_{2}=0$ and

$$
c=-h G+\frac{\alpha}{2} h^{2} .
$$

Proof. The only complication is in the $\Gamma$ equation, where the left-hand side has a constant term and a term linear in $\xi$. The restrictions on $K_{2}$ and $c$ come from equating coefficients.

Interestingly, the speed in (4.3) is the transport velocity in (2.4); it is neither the transport velocity $-\frac{1}{2} h G+\frac{\alpha}{3} h^{2}$ nor the characteristic speed $-h G+\alpha h^{2}$ for the conservation law (2.3) with $\Gamma_{x}=G$ constant. This is not a contradiction, since $h$ is constant.

There are also nonlinear traveling waves corresponding to solutions of system (4.1), (4.2) in which $h$ is not constant and $\Gamma$ is not linear. Consider a solution $h(\xi)$, $\Gamma(\xi)$ with boundary conditions at $\xi=-\infty$ :

$$
h(-\infty)=h_{L}, \quad \Gamma(-\infty)=\Gamma_{L}, \quad \Gamma^{\prime}(-\infty)=0 .
$$


These conditions serve to determine the constants $K_{1}, K_{2}$, so that (4.1), (4.2) become

$$
\begin{aligned}
& -c h-\frac{1}{2} h^{2} \Gamma^{\prime}+\frac{\alpha}{3} h^{3}=-c h_{L}+\alpha \frac{h_{L}^{3}}{3}, \\
& -c \Gamma-h \Gamma \Gamma^{\prime}+\frac{\alpha}{2} h^{2} \Gamma=-c \Gamma_{L}+\frac{\alpha}{2} h_{L}^{2} \Gamma_{L} .
\end{aligned}
$$

Eliminating $\Gamma^{\prime}$, we obtain an invariant curve through $\left(h_{L}, \Gamma_{L}\right)$ in the $(h, \Gamma)$-plane:

$$
\Gamma(h)=\frac{h \Gamma_{L}\left(c-\frac{\alpha}{2} h_{L}^{2}\right)}{c\left(2 h_{L}-h\right)+\alpha\left(\frac{1}{6} h^{3}-\frac{2}{3} h_{L}^{3}\right)} .
$$

On the curve $\Gamma(h)$, the flow is given by solving (4.1) for $\Gamma^{\prime}$ and using the chain rule $\frac{d \Gamma}{d \xi}=\frac{d \Gamma}{d h} \frac{d h}{d \xi}$ to obtain an expression for $h^{\prime}$. Differentiating (4.7), we obtain

$$
\frac{d \Gamma}{d h}=\frac{6 \Gamma_{L}\left(2 c-\alpha h_{L}^{2}\right)\left(6 c h_{L}-\alpha h^{3}-2 \alpha h_{L}^{3}\right)}{\left(12 c h_{L}-6 c h+\alpha h^{3}-4 \alpha h_{L}^{3}\right)^{2}} .
$$

Therefore, after some processing, we find

$$
\begin{gathered}
\frac{d \Gamma}{d \xi}=\frac{\frac{2}{3}\left(h_{L}-h\right)\left(3 c-\alpha\left(h^{2}+h h_{L}+h_{L}^{2}\right)\right)}{h^{2}} \\
\frac{d h}{d \xi}=\frac{-\frac{1}{9}\left(h-h_{L}\right)\left(3 c-\alpha\left(h^{2}+h h_{L}+h_{L}^{2}\right)\right)\left(12 c h_{L}-6 c h+\alpha h^{3}-4 \alpha h_{L}^{3}\right)^{2}}{h^{2} \Gamma_{L}\left(2 c-\alpha h_{L}^{2}\right)\left(6 c h_{L}-\alpha h^{3}-2 \alpha h_{L}^{3}\right)} .
\end{gathered}
$$

In particular, the point $\left(h_{L}, \Gamma_{L}\right)$ is an equilibrium; whether it is stable or unstable depends on the signs of the various factors in (4.9), (4.10). It is unstable for the traveling waves we seek.

As suggested by the numerical results of the previous section, the traveling waves of most interest to us in this paper have $h$ and $\Gamma$ increasing, with $h$ approaching finite limits at $\pm \infty$, and $\Gamma$ unbounded at $+\infty$. The following result establishes the existence of such waves.

THEOREM 4.2. For each $h_{M} \in\left(h_{L}, 2^{\frac{2}{3}} h_{L}\right)$, there is a traveling wave solution $h(\xi), \Gamma(\xi), \xi=x-$ ct satisfying

(i) $h^{\prime}(\xi)>0 ; \Gamma^{\prime}(\xi)>0$;

(ii) $h(-\infty)=h_{L}, \Gamma(-\infty)=\Gamma_{L}, h(\infty)=h_{M}, \Gamma(\infty)=\infty, \Gamma^{\prime}(\infty)=G_{M}$ with speed

$$
c=-h_{M} G_{M}+\frac{\alpha}{2} h_{M}^{2}
$$

where

$$
G_{M}=\frac{\alpha}{3} \frac{\left(h_{L}-h_{M}\right)}{h_{M}\left(2 h_{L}-h_{M}\right)}\left(h_{M}^{2}-2 h_{L} h_{M}-2 h_{L}^{2}\right) .
$$

Proof. Let

$$
d\left(h ; c, h_{L}\right)=c\left(2 h_{L}-h\right)+\frac{\alpha}{6}\left(h^{3}-4 h_{L}^{3}\right),
$$

the denominator in the expression (4.7) for $\Gamma=\Gamma(h)$. Solving $d\left(h ; c, h_{L}\right)=0$, we find

$$
c=c(h)=\frac{\alpha}{6}\left(\frac{h^{3}-4 h_{L}^{3}}{h-2 h_{L}}\right) .
$$


By considering the graph of this rational function of $h$, we find $c$ has a positive local maximum at $h=h_{L}$, a zero at $h=2^{\frac{2}{3}} h_{L}<2 h_{L}$, and $c(0)=\frac{\alpha}{3} h_{L}^{2}$. Consequently, for $h_{M}$ in the range of the theorem, there is a single zero of $d$, parameterized by either $h=h_{M}$ or by $c \in\left(0, \frac{\alpha}{2} h_{L}^{2}\right)$. For $\frac{\alpha}{3} h_{L}^{2}<c<\frac{\alpha}{2} h_{L}^{2}$, there is a second positive root $h_{0}<h_{L}$, which crosses $h=0$ at $c=\frac{\alpha}{3} h_{L}^{2}$.

In summary, the zero of $d$ that we seek is given by (4.13) with $h=h_{M}$ :

$$
c=c\left(h_{M}\right)=\frac{\alpha}{6}\left(\frac{h_{M}^{3}-4 h_{L}^{3}}{h_{M}-2 h_{L}}\right), \quad h_{L}<h_{M}<2^{\frac{2}{3}} h_{L} .
$$

Then $\Gamma(h)$ has a vertical asymptote at $h=h_{M}$ and, moreover, $\Gamma$ stays positive: $\Gamma(h) \rightarrow \infty$ as $h \nearrow h_{M}$. Examining the flow (4.9), (4.10), we find that $\left(h_{L}, \Gamma_{L}\right)$ is an unstable equilibrium. Let $(h(\xi), \Gamma(\xi))$ be the corresponding trajectory satisfying (4.5), (4.6) with $(h(-\infty), \Gamma(-\infty))=\left(h_{L}, \Gamma_{L}\right), h^{\prime}>0, \Gamma^{\prime}>0$. Then $h(\infty)=h_{M}$ and $\Gamma(\infty)=\infty$.

The final step is to set $h=h_{M}$ in (4.9), from which we find (after substitution for $c$ from (4.14)) $\Gamma^{\prime}(\xi) \rightarrow G_{M}$, as given in (4.12). Formula (4.11) is then easily checked.

Remarks. 1. Formula (4.11) suggests that the traveling wave approaches a simple traveling wave as $\xi \rightarrow \infty$. Indeed, $h(\xi) \rightarrow h_{M}$, a constant, and $\Gamma^{\prime}(\xi) \rightarrow G_{M}$, as $\xi \rightarrow \infty$.

2. Equations (4.11), (4.12) link the asymptotic behavior as $\xi \rightarrow \infty$ to the equilibrium $\left(h_{L}, \Gamma_{L}\right)$ without needing to integrate the ODEs. The formulae are independent of $\Gamma_{L}$, reflecting the scale invariance of the equations. Moreover, these formulae persist as $\Gamma_{L} \rightarrow 0$. In this limit, the traveling wave approaches a simple nonsmooth wave (see Corollary 5.5 below).

3. From (4.14), we observe that $c\left(h_{M}\right) \in\left(\frac{\alpha}{3} h_{L}^{2}, \frac{\alpha}{2} h_{L}^{2}\right)$, an interval that collapses onto zero as $\alpha \rightarrow 0$. Consequently, the nonlinear traveling waves of the theorem do not appear in the case $\alpha=0$ of the horizontal substrate.

5. Jump conditions. As discussed above, and as observed in numerical simulations, solutions of system (2.3), (2.4) typically contain discontinuities in $h$ and $\Gamma_{x}$. In this section, we begin a systematic study of the Rankine-Hugoniot jump conditions for the system.

Consider the Cauchy problem for system (2.3), (2.4) with initial data

$$
h(x, 0)=h_{0}(x), \quad \Gamma(x, 0)=\Gamma_{0}(x), \quad-\infty<x<\infty .
$$

By a weak solution of the Cauchy problem, we mean a function $(h, \Gamma): \mathbb{R} \times \mathbb{R}_{+} \rightarrow \mathbb{R}_{+}^{2}$ with $h, \Gamma, \Gamma_{x} \in L^{\infty} \cap L_{l o c}^{1}$ such that for every test function $\phi \in C_{c}^{\infty}\left(\mathbb{R} \times \mathbb{R}_{+}\right)$,

$$
\begin{aligned}
\int_{0}^{\infty} \int_{-\infty}^{\infty}\left\{h \phi_{t}-\left(\frac{1}{2} h^{2} \Gamma_{x}-\frac{\alpha}{3} h^{3}\right) \phi_{x}\right\} d x d t & =\int_{-\infty}^{\infty} h_{0} \phi(x, 0) d x \\
\int_{0}^{\infty} \int_{-\infty}^{\infty}\left\{\Gamma \phi_{t}-\left(h \Gamma \Gamma_{x}-\frac{\alpha}{2} h^{2} \Gamma\right) \phi_{x}\right\} d x d t & =\int_{-\infty}^{\infty} \Gamma_{0} \phi(x, 0) d x .
\end{aligned}
$$

Note that in this definition of weak solutions, $\Gamma(x, t)$ is continuous with respect to $x$ for each time $t$.

In analyzing jump conditions, we will always assume that $h$ is $C^{1}$ and $\Gamma$ is $C^{2}$, apart from a finite number of curves $x=\gamma(t)$ across which $h$ and $\Gamma_{x}$ can have jump discontinuities. We refer to such solutions as piecewise-smooth. We shall be 
particularly interested in solutions in which surfactant spreads into a region with initially uniform film height. Let $(h, \Gamma)$ be a piecewise-smooth weak solution, and suppose the leading edge of the surfactant is located on a curve $x=\gamma_{\ell}(t)$ :

$$
\Gamma(x, t)=0, \quad x \geq \gamma_{\ell}(t) .
$$

The following theorem determines the speed at the leading edge of the surfactant.

THEOREM 5.1. Let $h, \Gamma$ be a piecewise-smooth weak solution of (2.3), (2.4) satisfying (5.3). If $G_{-} \equiv \Gamma_{x}\left(\gamma_{\ell}(t)-, t\right)<0$, then

$$
\gamma_{\ell}^{\prime}(t)=-h_{-} G_{-}+\frac{\alpha}{2} h_{-}^{2},
$$

where $h_{-}=h\left(\gamma_{\ell}(t)-, t\right)$.

Proof. Differentiating $\Gamma\left(\gamma_{\ell}(t)-, t\right)=0$, we have

$$
\Gamma_{x} \gamma_{\ell}^{\prime}(t)+\Gamma_{t}=0 .
$$

But from (2.4), $\Gamma_{t}=\left(h \Gamma \Gamma_{x}\right)_{x}-\frac{\alpha}{2}\left(h^{2} \Gamma\right)_{x}=h_{-} G_{-}^{2}-\frac{\alpha}{2} h_{-}^{2} G_{-}$, since $\Gamma=0$ at the leading edge $x=\gamma_{\ell}(t)$. The formula (5.4) now follows, since $\Gamma_{x}=G_{-} \neq 0$.

Now we derive a set of equations from the Rankine-Hugoniot conditions for the discontinuities in $h$ and $\Gamma_{x}$ and an equation enforcing the continuity of $\Gamma$ at the discontinuities. Consider a solution $(h, \Gamma)$ that is smooth away from a differentiable curve $C=\{x=\gamma(t)\}$ such that $\Gamma$ is continuous across $C$, and $h, \Gamma_{x}$ have well-defined one-sided limits at $C$. Let $[u]$ denote the jump in a function $u$ across $C$, and let $c=\gamma^{\prime}(t)$. It is also convenient to use the notation

$$
G=\Gamma_{x}, \quad \bar{\Gamma}=\Gamma(\gamma(t), t) .
$$

Then the jump conditions are as follows. From (2.3) we obtain

$$
-c[h]+\left[-\frac{1}{2} h^{2} G+\frac{\alpha}{3} h^{3}\right]=0
$$

while (since $\Gamma$ is continuous) (2.4) yields

$$
\bar{\Gamma}\left[-h G+\frac{\alpha}{2} h^{2}\right]=0 .
$$

The continuity of $\Gamma$ provides an additional equation matching the left and right limits of $\Gamma$ at $x=\gamma(t)$ :

$$
\Gamma(\gamma(t)-, t)=\Gamma(\gamma(t)+, t) .
$$

We refer to weak solutions that consist of a simple traveling wave (see section 4) on either side of a jump in $h, \Gamma_{x}$ as a simple jump. In a simple jump, both $h$ and $G$ are constant, so that the jump condition (5.5) implies the speed is constant also. By translation invariance, we may without loss of generality take the simple jump to lie along the line $x=c t$ :

$$
h(x, t)=\left\{\begin{array}{ll}
h_{-} & \text {if } x<c t, \\
h_{+} & \text {if } x>c t,
\end{array} \quad \Gamma(x, t)= \begin{cases}\Gamma_{0}+G_{-}\left(x-c_{-} t\right) & \text { if } x<c t \\
\Gamma_{0}+G_{+}\left(x-c_{+} t\right) & \text { if } x>c t\end{cases}\right.
$$


In this solution, we are leaving open the possibility that the simple traveling waves on either side of the jump travel with different speeds. In this case, continuity of $\Gamma$ (5.7) requires

$$
G_{-}\left(c-c_{-}\right)=G_{+}\left(c-c_{+}\right) .
$$

The jump conditions (5.5), (5.6) for a simple jump (5.8) become

$$
\begin{aligned}
-c\left(h_{+}-h_{-}\right)-\frac{1}{2}\left(h_{+}^{2} G_{+}-h_{-}^{2} G_{-}\right)+\frac{\alpha}{3}\left(h_{+}^{3}-h_{-}^{3}\right) & =0, \\
\left(\Gamma_{0}+G_{-}\left(c-c_{-}\right) t\right)\left(-h_{+} G_{+}+h_{-} G_{-}+\frac{\alpha}{2}\left(h_{+}^{2}-h_{-}^{2}\right)\right) & =0 .
\end{aligned}
$$

Equating coefficients in the second equation, we have a pair of conditions:

$$
\begin{aligned}
\Gamma_{0}\left(-h_{+} G_{+}+h_{-} G_{-}+\frac{\alpha}{2}\left(h_{+}^{2}-h_{-}^{2}\right)\right) & =0, \\
G_{-}\left(c-c_{-}\right)\left(-h_{+} G_{+}+h_{-} G_{-}+\frac{\alpha}{2}\left(h_{+}^{2}-h_{-}^{2}\right)\right) & =0 .
\end{aligned}
$$

The analysis of the jump conditions is organized as follows. First, we show that for $\alpha=0$, and $\bar{\Gamma}=0$, there is a simple jump in $h$; the height doubles across the jump, and the speed is determined by the height and surfactant concentration gradient on one side of the jump. However, when $\bar{\Gamma}>0$, there are no simple jumps when $\alpha=0$. For $\alpha>0$, there are two simple jumps: one at the leading edge, but with the jump in height coupled to the surfactant concentration gradient, and another in the bulk, where the jump conditions can be solved explicitly.

The horizontal substrate: $\boldsymbol{\alpha}=\mathbf{0}$. First, we investigate the structure of the leading edge of the surfactant when $\alpha=0$. The speed $c=\gamma_{\ell}^{\prime}(t)$ of the leading edge $x=\gamma_{\ell}(t)$ was characterized in Theorem 5.1.

TheOREm $5.2 .^{2}$ Let $h, \Gamma$ be a piecewise-smooth weak solution of (2.3), (2.4) with $\alpha=0$ satisfying (5.3). If $G_{-}=\Gamma_{x}\left(\gamma_{\ell}(t)-, t\right)<0$, then

$$
h_{-}=2 h_{+},
$$

where $h_{ \pm}=h\left(\gamma_{\ell}(t) \pm, t\right)$.

Proof. The result follows immediately by substituting the expression for the speed at the jump (5.4) into the height jump condition (5.5). Specifically, (5.5) with $\alpha=0$ becomes

$$
-c\left(h_{+}-h_{-}\right)+\frac{1}{2} h_{-}^{2} G_{-}=0,
$$

since $G_{+}=\Gamma_{x}\left(\gamma_{\ell}(t)+, t\right)=0$. But from (5.4), $c=-h_{-} G_{-}$. Substitution into (5.14) leads to the result, since $G_{-} \neq 0$.

Next, we show there are no simple jumps with $\bar{\Gamma}>0$.

TheOrem 5.3. Let $\alpha=0, \bar{\Gamma}>0$. There are no simple jumps with $c \neq 0$. For $c=0$, there is a nonphysical solution with a stationary jump in $h$ and no jump in $\Gamma_{x}$.

Proof. Set $\alpha=0$ in the jump conditions (5.10), (5.12) to get

$$
-c\left(h_{+}-h_{-}\right)-\frac{1}{2} h_{+}^{2} G_{+}+\frac{1}{2} h_{-}^{2} G_{-}=0
$$

\footnotetext{
${ }^{2}$ These jumps were first characterized by Borgas and Grotberg [3].
} 
and

$$
\begin{array}{r}
\Gamma_{0}\left(-h_{+} G_{+}+h_{-} G_{-}\right)=0, \\
G_{-}\left(c-c_{-}\right)\left(-h_{+} G_{+}+h_{-} G_{-}\right)=0 .
\end{array}
$$

Since $\Gamma_{0}>0$, we see that

$$
h_{-} G_{-}=h_{+} G_{+} .
$$

Consequently, the speeds $c_{-}, c_{+}$of the simple traveling waves on each side of the simple jump are equal (see Theorem 4.1 with $\alpha=0$ ):

$$
c_{-}=-h_{-} G_{-}=c_{+}=-h_{+} G_{+} .
$$

Substituting (5.17) into (5.15), we conclude that either $h_{+}=h_{-}$, in which case $G_{+}=G_{-}$and there is no jump, or $h_{+} \neq h_{-}$and a jump in $h$ and $G$ would have speed

$$
c=-\frac{1}{2} h_{-} G_{-} .
$$

But then (5.9), (5.18) imply that either $c=c_{-}=c_{+}=0$, so that $G_{-}=G_{+}=0$, or $G_{-}=G_{+} \neq 0$. In the former case, we regard the solution with an arbitrary stationary jump in $h$ as unphysical, since the fluid discontinuity would collapse in the presence of additional smoothing terms such as capillarity or second order diffusion. In the latter case, (5.17) implies $h_{-}=h_{+}$, and we are back to the simple traveling wave of the previous section with no jump in $h$ or $\Gamma_{x}$.

The inclined substrate: $\boldsymbol{\alpha}>\mathbf{0}$. When we consider a film flowing down an inclined substrate in which $\alpha>0$, we find solutions of the jump conditions that are strikingly different from the $\alpha=0$ case. We explore the wave structures analytically here and numerically in section 7 . As with $\alpha=0$, we treat the leading edge of surfactant separately.

THEOREM 5.4. Let $h, \Gamma$ be a piecewise-smooth weak solution of (2.3), (2.4) with $\alpha>0$ satisfying (5.3). Let $h_{ \pm}=h\left(\gamma_{\ell}(t) \pm, t\right)$, and suppose $G_{-}=\Gamma_{x}\left(\gamma_{\ell}(t)-, t\right)<0$. Then

$$
\text { (a) } h_{-}<h_{+} \text {or (b) } 2 h_{+}<h_{-}<(1+\sqrt{3}) h_{+} \text {; }
$$

in both cases

$$
G_{-}=\frac{\alpha}{3} \frac{\left(h_{-}-h_{+}\right)\left(h_{-}^{2}-2 h_{-} h_{+}-2 h_{+}^{2}\right)}{h_{-}\left(h_{-}-2 h_{+}\right)}, \quad \gamma_{\ell}^{\prime}(t)=-h_{-} G_{-}+\frac{\alpha h_{-}^{2}}{2} .
$$

Proof. The speed in (5.21) is given by Theorem 5.1 (see (5.4)). Substituting $c=\gamma_{\ell}^{\prime}(t)$ and $G_{+}=0$ into the jump condition (5.10) gives the formula for $G_{-}$in (5.21). The inequalities (5.20) are equivalent to $G_{-}<0$. (The inequality $G_{-} \leq 0$ is needed to ensure $\Gamma>0$ behind the leading edge of the surfactant.)

Remarks. 1. For $\alpha>0$, the jump in $h$ and the surfactant concentration gradient are linked, in contrast to the $\alpha=0$ case, in which the jump in $h$ is determined, and $G_{-}$is a free parameter that influences only the speed.

2. Conditions (5.21) correspond to the limit $\Gamma_{L} \rightarrow 0$ of the nonlinear traveling wave in Theorem 4.2 .

3. The case $h_{-}<h_{+}$appears to be unphysical; both gravity and the surfactant concentration gradient act downwards and cannot sustain such a jump in the height. 
We conjecture that this jump is unstable, as suggested by the numerical evidence in Figure 8. Further explanation for the instability of the wave is suggested by the observation that the speed of the discontinuity in (5.21) is smaller than the characteristic speed $\alpha h_{+}^{2}$ in the surfactant-free region ahead of the discontinuity.

The following corollary parallels the result of Theorem 5.4, except that it concerns the trailing edge of the surfactant, so the roles of $h_{+}, h_{-}$are exchanged. However, the cases are not symmetric, since gravity and the Marangoni force are now opposed. In particular, $h_{+}>h_{-}$.

Corollary 5.5. Let $h, \Gamma$ be a piecewise-smooth weak solution of (2.3), (2.4) with $\alpha>0$ satisfying $\Gamma(x, t)=0, x \leq \gamma_{T}(t)$. Let $h_{ \pm}=h\left(\gamma_{T}(t) \pm, t\right)$, and suppose $G_{+}=\Gamma_{x}\left(\gamma_{T}(t)+, t\right)>0$. Then

$$
\text { (a) } h_{+}>(1+\sqrt{3}) h_{-} \text {or (b) } h_{-}<h_{+}<2 h_{-} \text {; }
$$

in both cases

$$
G_{+}=\frac{\alpha}{3} \frac{\left(h_{+}-h_{-}\right)\left(h_{+}^{2}-2 h_{-} h_{+}-2 h_{-}^{2}\right)}{h_{+}\left(h_{+}-2 h_{-}\right)}, \quad \gamma_{T}^{\prime}(t)=-h_{+} G_{+}+\frac{\alpha h_{+}^{2}}{2} .
$$

Proof. The proof parallels that of Theorem 5.4 with $h_{-}, h_{+}$switched; the bounds on $h_{ \pm}$ensure $G_{+}>0$.

THEOREM 5.6. For $\alpha>0$, let $h, \Gamma$ be a piecewise-smooth weak solution of (2.3), (2.4) with a simple jump across the line $x=c t$, where $\Gamma=\Gamma_{0}>0$. Then either

(a) $\Gamma=\Gamma_{0}+G(x-s t)$ is linear, in which case

$$
G=\frac{\alpha}{2}\left(h_{+}+h_{-}\right) ; \quad s=-\frac{\alpha}{2} h_{+} h_{-} ; \quad c=\frac{\alpha}{12}\left(h_{+}-h_{-}\right)^{2},
$$

or (b) $\Gamma_{x}$ has a jump with

$$
\begin{gathered}
G_{-}=-\frac{\alpha}{6 h_{-}}\left(h_{+}-h_{-}\right)\left(h_{+}+2 h_{-}\right) ; \quad G_{+}=\frac{\alpha}{6 h_{+}}\left(h_{+}-h_{-}\right)\left(2 h_{+}+h_{-}\right) ; \\
c=\frac{\alpha}{6}\left(h_{+}^{2}+h_{+} h_{-}+h_{-}^{2}\right) .
\end{gathered}
$$

Proof. In case (a), we substitute $\Gamma=\Gamma_{0}+G(x-s t), h=h_{ \pm}$into (2.4) to find

$$
s=-h_{+} G+\frac{\alpha}{2} h_{+}^{2}=-h_{-} G+\frac{\alpha}{2} h_{-}^{2} .
$$

(That is, $\left(h_{ \pm}, \Gamma\right)$ is a simple traveling wave with the same speed $s$ and surfactant gradient $G$ on each side of $x=c t$ but with a jump in $h$.) The second equality gives the formula for $G$ (consistent with (5.6)) from which the expression for $s$ follows. The speed of the jump in $h$ comes from the jump condition (5.10) in which $G_{ \pm}=G$.

In case (b), since $\Gamma>0$, the jump condition (5.11) together with Theorem 4.1 for simple traveling waves implies

$$
c_{+}=-h_{+} G_{+}+\frac{\alpha}{2} h_{+}^{2}=-h_{-} G_{-}+\frac{\alpha}{2} h_{-}^{2}=c_{-} .
$$

But now continuity of $\Gamma$ (see (5.9)) and $G_{+} \neq G_{-}$imply $c=c_{+}=c_{-}$. Substituting into the jump condition (5.10) leads to the result (noting that $h_{+}=h_{-}$implies $\left.G_{+}=G_{-}\right)$.

Remark. The motion of the film in this case is somewhat surprising, since the linear wave with slope $G=\frac{\alpha}{2}\left(h_{+}+h_{-}\right)$moves to the left with speed $s=-\frac{\alpha}{2} h_{+} h_{-}$, while simultaneously the jump in height moves to the right with speed $c=\frac{\alpha}{12}\left(h_{+}-h_{-}\right)^{2}$. 
6. Combining waves when $\boldsymbol{\alpha}>\boldsymbol{0}$. The numerical experiments of section 3 suggest that as time increases, the solution approaches a combination of traveling waves and simple jumps. We can now interpret this structure in terms of the traveling waves of section 4 and the jumps of section 5. Each of the four numbered features in the figure is associated with formulae relating the parameters labeled in the figure to each other and to the wave speeds. In processing the equations, we observe that the jump condition (5.6) effectively equates wave speeds of simple traveling waves on either side of a jump. Consequently, the four wave speeds are all equal to a single speed $c$. We combine the following:

(1) A traveling wave with speed $c$ connecting $h_{L}$ to $h_{M}$ (see Theorem 4.2):

$$
\begin{gathered}
c=\frac{\alpha\left(\frac{1}{6} h_{M}^{3}-\frac{2}{3} h_{L}^{3}\right)}{h_{M}-2 h_{L}}, \\
G_{M}=\frac{\alpha}{3} \frac{\left(h_{L}-h_{M}\right)\left(h_{M}^{2}-2 h_{L} h_{M}-2 h_{L}^{2}\right)}{h_{M}\left(2 h_{L}-h_{M}\right)} .
\end{gathered}
$$

(2) A simple jump in $h, \Gamma_{x}$ (see Theorem 5.6):

$$
\begin{aligned}
G_{M} & =-\frac{\alpha}{6 h_{M}}\left(h_{m}-h_{M}\right)\left(h_{m}+2 h_{M}\right), \\
G_{m} & =\frac{\alpha}{6 h_{m}}\left(h_{m}-h_{M}\right)\left(2 h_{m}+h_{M}\right) .
\end{aligned}
$$

(3) A simple traveling wave with $\Gamma$ descending to zero and (4) a simple jump in $h$ (see Theorems 4.1 and 5.4):

$$
G_{m}=\frac{\alpha}{3} \frac{\left(h_{m}-h_{R}\right)\left(h_{m}^{2}-2 h_{m} h_{R}-2 h_{R}^{2}\right)}{h_{m}\left(h_{m}-2 h_{R}\right)} .
$$

Noting the symmetry in the expressions for $G_{m}$ and $G_{M}$, we solve the equations by treating $h_{L}$ and $h_{R}$ as parameters. We can then simplify the equations by equating $G_{M}$ in (6.2), (6.3) and equating $G_{m}$ in (6.4), (6.5), giving two simultaneous equations for $h_{M}, h_{m}$ :

$$
\begin{aligned}
& 2 h_{L}\left(h_{m}^{2}+h_{M}^{2}-2 h_{L}^{2}\right)-h_{M} h_{m}\left(h_{M}+h_{m}-2 h_{L}\right)=0, \\
& 2 h_{R}\left(h_{m}^{2}+h_{M}^{2}-2 h_{R}^{2}\right)-h_{M} h_{m}\left(h_{M}+h_{m}-2 h_{R}\right)=0 .
\end{aligned}
$$

THEOREM 6.1. The polynomial equations (6.6), (6.7) have two positive solutions for $0<h_{R} / h_{L}<r^{*}$, where $r^{*}=\frac{1}{2}(\sqrt{3}-1)$, and no solution for $r^{*}<h_{R} / h_{L}<1$. The only relevant solution has $h_{m}<h_{M}$. (6.7):

Proof. It is perhaps easier to see the structure if we rewrite the variables in (6.6),

$$
x=h_{M}, \quad y=h_{m}, \quad u=h_{L}, \quad v=h_{R} .
$$

Then the system becomes

$$
\begin{aligned}
& 2 u\left(x^{2}+y^{2}-2 u^{2}\right)-x y(x+y-2 u)=0, \\
& 2 v\left(x^{2}+y^{2}-2 v^{2}\right)-x y(x+y-2 v)=0 .
\end{aligned}
$$


Since $u \neq v$, taking $v(\mathrm{a})-u(\mathrm{~b})$ leads to

$$
x y(x+y)=4 u v(u+v) .
$$

Substituting back into (6.8(a)), we get

$$
x^{2}+y^{2}+x y=2\left(u^{2}+u v+v^{2}\right) .
$$

We can eliminate $x$, since $x y(x+y)=y\left(x^{2}+x y\right)$. Thus, $x^{2}+x y=4 u v(u+v) / y$. Substituting into (6.10),

$$
y^{2}+4 u v(u+v) / y=2\left(u^{2}+u v+v^{2}\right),
$$

leaving a cubic in $y$ :

$$
y^{3}-a y+b=0,
$$

where $a=2\left(u^{2}+u v+v^{2}\right), b=4 u v(u+v)$. Consequently, the equation has one negative root and zero, one, or two positive roots, since $u>0, v>0$. The number of solutions of (6.11) changes from one to three precisely on the curve

$$
27 b^{2}=4 a^{3} .
$$

In terms of $u, v$ this equation is

$$
27 u^{2} v^{2}(u+v)^{2}=2\left(u^{2}+u v+v^{2}\right)^{3} .
$$

The polynomial $27 u^{2} v^{2}(u+v)^{2}-2\left(u^{2}+u v+v^{2}\right)^{3}$ has three quadratic factors:

$$
\begin{aligned}
& 27 u^{2} v^{2}(u+v)^{2}-2\left(u^{2}+u v+v^{2}\right)^{3} \\
& \quad=\left(v^{2}+4 u v+u^{2}\right)\left(v^{2}-2 u v-2 u^{2}\right)\left(u^{2}-2 u v-2 v^{2}\right) .
\end{aligned}
$$

The first factor is positive in the first quadrant, and the other factors have conjugate roots stemming from

$$
\frac{v}{u}=\frac{1}{2}(\sqrt{3}-1) \equiv r^{*},
$$

the threshold beyond which we can no longer obtain solutions consistent with (6.1) through (6.5).

To understand better how the various parameters relate to each other, first we note that $x=h_{m}$ and $y=h_{M}$ are interchangeable in (6.8). Without loss of generality, we take $u=h_{L}=1$ and rewrite (6.11) as a quadratic equation for $v$ in terms of $y=h_{m}$ $\left(\right.$ or $\left.h_{M}\right)$ :

$$
v^{2}+v+\frac{y\left(y^{2}-2\right)}{2(2-y)}=0
$$

For $0<y<\sqrt{2}$, the product of the two roots is negative, so they are real and of opposite sign. In Figure 6, we plot $v=h_{R}$ on the horizontal axis to clarify that for each choice of $h_{R}, 0<h_{R}<r^{*}$, there are two values of $y$ corresponding to $h_{m}, h_{M}$. From this graph or from (6.13), we see that, as the precursor height $h_{R}=v \rightarrow 0$, we have $h_{m} \rightarrow 0$ and $h_{M} \rightarrow \sqrt{2}$. From (6.3), we find that $G_{m} \rightarrow-\infty, G_{M} \rightarrow \frac{\sqrt{2}}{3}$. 


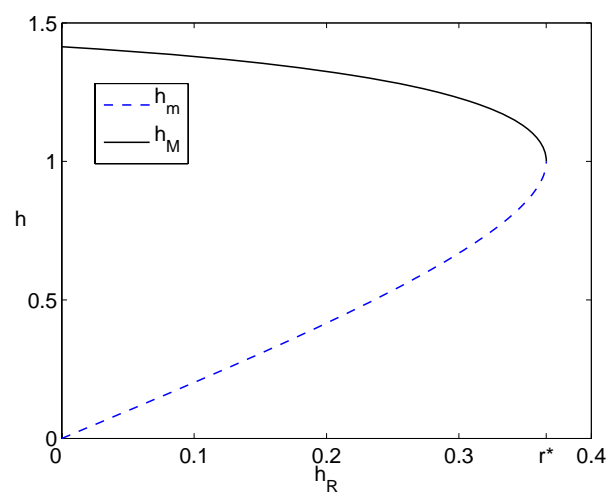

FIG. 6. Plot of $h_{R}$ versus $h_{m}, h_{M}$ from (6.13).

Correspondingly, the speed $c \rightarrow \frac{1}{3}$, a value that appears in the proof of Theorem 4.2. That is, if $h_{M}$ is restricted to lie in the interval $\left(h_{L}, \sqrt{2} h_{L}\right)$ in Theorem 4.2, then the traveling wave speed is in the interval $\left(\frac{\alpha}{3} h_{L}^{2}, \frac{\alpha}{2} h_{L}^{2}\right)$. In Figure 6 we observe that $h_{m}, h_{M}$ both approach $h=1$ as $v=h_{R}$ approaches the threshold $r^{*}=\frac{1}{2}(\sqrt{3}-1)$ of Theorem 6.1.

For a film propagating onto a prewetted surface (i.e., with a small precursor height $h_{R}$ ), it might seem reasonable from Figure 6, relating the values of $h_{M}$ and $h_{m}$ to $h_{R}$, to approximate the height of the step to be twice that of the precursor height. However, for larger precursors this is not a good approximation. The step is always greater than twice the precursor height; in fact, for a given $h_{R}$, as $h_{m}$ approaches $2 h_{R}, G_{M}$ approaches $\infty$ and the speed of the wave goes to $\infty$. Moreover, $h_{m} \rightarrow 1$ as $h_{R} \rightarrow r^{*}$.

In the next section, numerical simulations illustrate the wave structures introduced in the analysis of sections 5 and 6 .

7. Numerical simulations II. PDE simulations of (2.3), (2.4) for the inclined substrate illustrate a number of issues presented in the above analysis. First, we explore a combination of linear waves and simple jumps using the analysis of section 6 to choose appropriate initial film profiles. Then we test the stability of linear waves and simple jumps, presented in Theorems 5.4 and 5.6. Next, we explore simulations near the critical threshold $r^{*}$. Finally, we vary $h_{R}$ to observe changes in the wave structures that occur above the threshold.

PDE simulations for linear waves and simple jumps. Equations (6.1)(6.5) were derived to explain the numerical simulations shown in Figure 4. However, the same equations apply to a combination of linear waves and simple jumps with $\Gamma_{L}=0=\Gamma_{R}$, all traveling with speed $c$ given by (6.1). The connection between the two structures stems from the limit $\Gamma_{L} \rightarrow 0$ in the nonlinear traveling wave, which does not affect the formulae in (6.1), (6.2).

In the PDE simulations of Figure 7, we choose initial data in which $h$ is piecewise constant and $\Gamma$ is piecewise linear, consistent with (6.1)-(6.5). The intermediate parameters $h_{m}, h_{M}, G_{m}$, and $G_{M}$ annotated in Figure 2 are calculated from (6.6), (6.7), (6.3), and (6.4) for the most common boundary conditions in the simulations, 

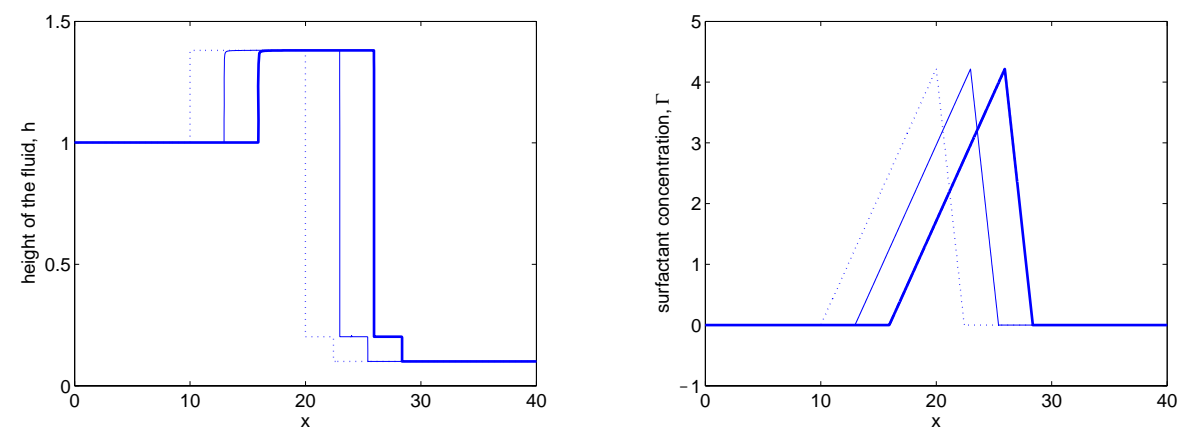

FIG. 7. PDE simulations of simple traveling waves. Initial profiles are dashed, final profiles are in bold, and plots are separated by 8 time units.
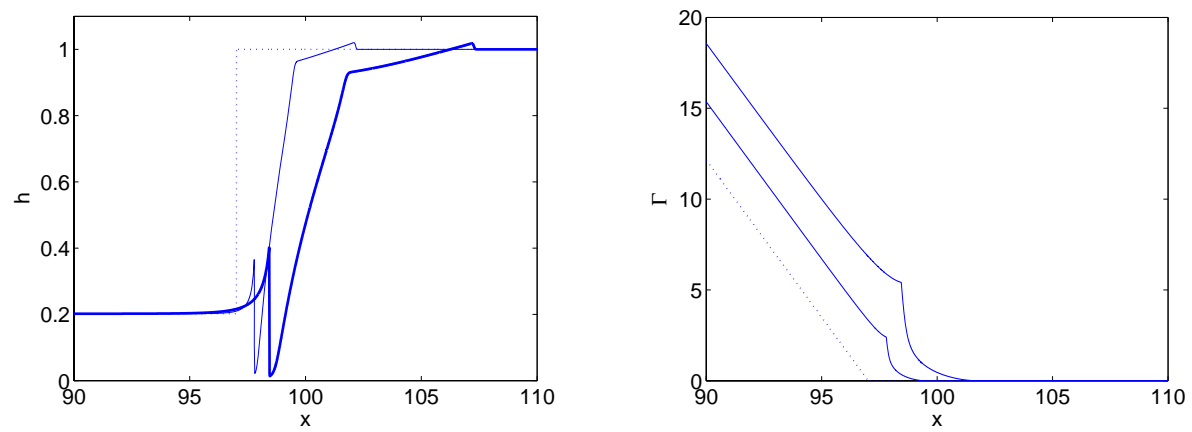

FIG. 8. Numerical evidence that the linear wave and jump combination of (5.20) with $h_{-}=0.2$, $h_{+}=1.0$ is unstable as in Theorem 5.4. Initial data are dotted, and final profiles in bold are at time $t=10$.

$h_{L}=1.0, h_{R}=0.1:$

$$
h_{M}=1.3787 ; \quad h_{m}=0.2019 ; \quad G_{M}=0.4210 ; \quad G_{m}=-1.7316 .
$$

The initial data have jumps at $x=10, x=20$; the initial location of the leading edge of the surfactant is then determined by the data.

In Figure 7, the entire structure moves to the right with speed 0.37 predicted by Theorem 5.4. Note that the maximum value of $\Gamma$ remains constant, as expected.

Figure 8 provides numerical evidence that the waves conjectured to be unstable in Theorem 5.4 are indeed so. In these plots notice that for the wave emerging from $h_{L}$, it appears that $h_{x} \rightarrow \infty$, followed by a jump in the height corresponding to a corner in $\Gamma_{x}$. To the right of the discontinuity, there is a smooth wave preceded by a hydrodynamic wave where there is no surfactant present.

The numerical experiments shown in Figures 9 and 10 explore the wave structure introduced in Theorem 5.6(a). In this case, the positive surfactant gradient creates a linear profile for $\Gamma$ that travels up the incline and maintains the jump in film height, which can have either sign. The depth-averaged velocity $-\frac{1}{2} h G+\frac{\alpha}{3} h^{2}$ changes sign across the jump, but the surface velocity $s=-\frac{\alpha}{2} h_{+} h_{-}$is negative, while the jump speed $c=\frac{\alpha}{12}\left(h_{+}-h_{-}\right)^{2}$ is positive. Since $G=\Gamma_{x}$ is constant, the jump in $h$ is a shock wave solution of (2.3). In general, a jump down satisfies the Lax entropy condition 

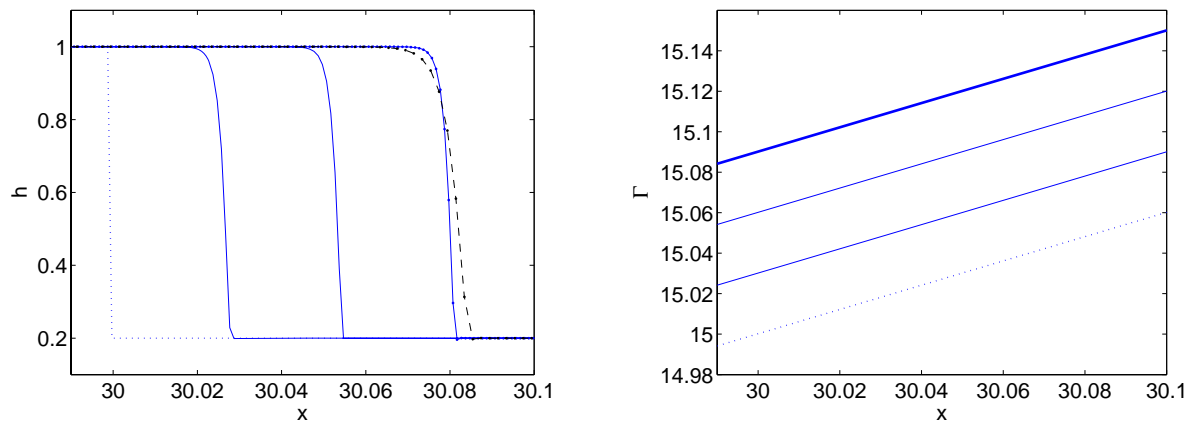

FIG. 9. PDE simulation of linear wave in $\Gamma$ moving to the left as jump in $h$ moves to the right. The initial condition is dotted, and the final profile at time $t=1.5$ is in bold $(\Delta x=.00025)$. For the height plot, the dashed profile at $t=1.5$ has a more coarse grid $(\Delta x=.001)$; the consistent location of grid points in the final profile at both mesh sizes and steepening with mesh refinement indicate the presence of a shock.
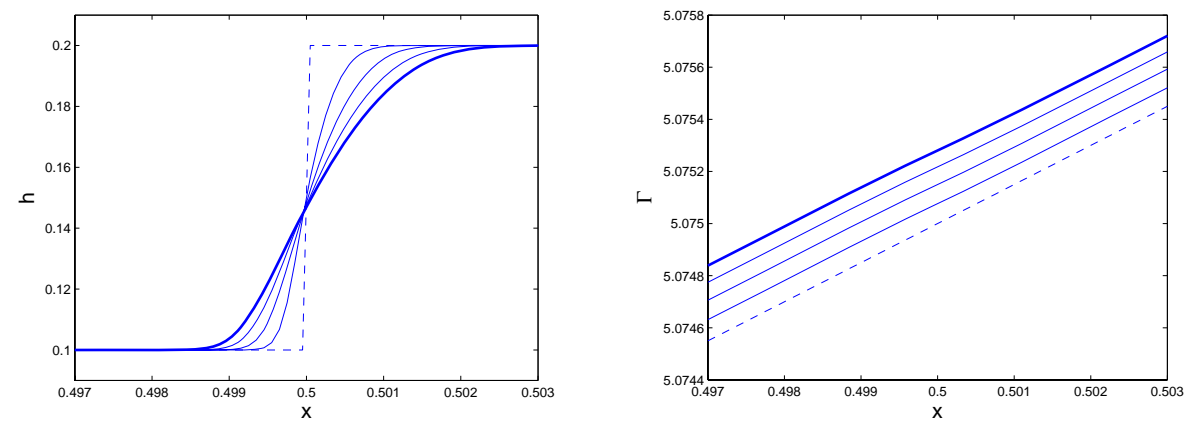

FIG. 10. PDE simulation of initially linear $\Gamma$ with a jump up in $h$ demonstrating the instability of jump-up shocks.

and is stable, as in Figure 9, while a jump up is unstable, as shown in Figure 10. In the latter case, it is tempting to think of the spreading solution as a rarefaction, with $\Gamma_{x}$ constant, but, in fact, the rarefaction solution fails to satisfy the surfactant equation (2.4).

PDE simulations varying $\boldsymbol{h}_{\boldsymbol{R}}$. Figure 11 contains the results of numerical simulation of the PDE with $h_{L}=1$ and with $h_{R}$ just above and just below the threshold of the theorem. For $h_{R}=0.365$, below the threshold, the step in $h$ is clearly emerging, but for $h_{R}=0.367$, above the threshold, the step fails to emerge. To quantify this observation, in Figure 12 we plot the difference in height $\Delta h$ between the local maximum near the leading shock and the local minimum immediately behind. Plotted as a function of time in the figure, we observe that $\Delta h$ approaches zero for $h_{R}=0.365$, whereas $\Delta h$ appears to be converging to a positive value for $h_{R}=0.367$.

Numerical PDE simulations with $h_{L}=1, \Gamma_{L}=1$, and $\alpha=1$ and varying values of $h_{R}$ below the critical threshold $h_{R}=\frac{\sqrt{3}-1}{2}$ of Theorem 6.1 reproduce the same pattern of waves shown in Figure 4. In Figure 13 we show the results of PDE simulations for selected values of $h_{R}$ above the critical threshold. These wave structures have not been observed previously, since $h_{R}$ is generally taken to be the thickness of a precursor 

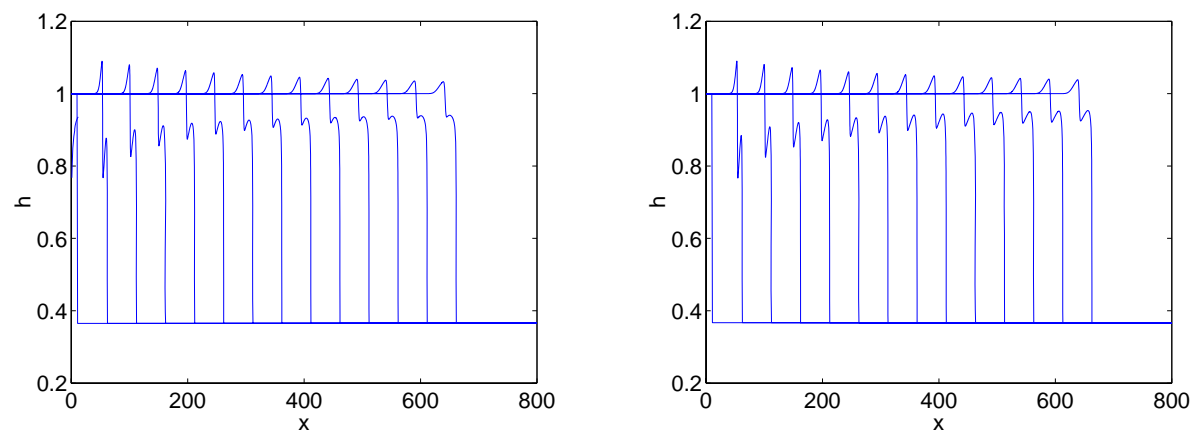

FIG. 11. Solutions near the transition between solution types. $h_{L}=1, h_{R}=0.365$ (left); $h_{L}=1, h_{R}=0.367$ (right). Plots are separated by 100 time units.

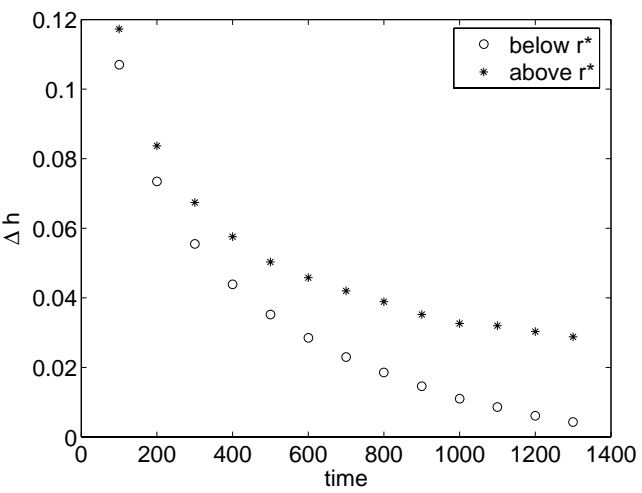

FIG. 12. Comparison of step heights for the plots in Figure 11. As the step forms for the solution below the critical threshold $\left(h_{R}<r^{*}\right)$, the difference $\Delta h$ in maximum and minimum heights within the step goes to zero. Above the threshold $\left(h_{R}>r^{*}\right)$ the height difference decreases much more slowly, evidence that no step emerges.

layer, and therefore much smaller than $h_{L}$. In all of the height plots, a distinctive jog in the height develops, which on the incline resembles a $Z$-shaped wave. We call this new wave a $Z$-wave, in which a shock is between two smooth waves. By comparison, an $N$-wave of hyperbolic conservation laws consists of a rarefaction bounded by two shocks. The middle height to the right of the $Z$ is the same as $h_{L}$, instead of the step height $h_{m}$ observed below the threshold.

In Figure 13(a), we observe a leading shock (i.e., a jump in $h$ ) ahead of the leading edge of surfactant. The shock is a solution of the conservation law

$$
h_{t}+\frac{1}{3}\left(h^{3}\right)_{x}=0,
$$

i.e., (2.3) with $\alpha=1$ and $\Gamma=0$. Similarly, in Figure 13(b), the fluid surface is initially flat. The surface tension gradient and gravity force a volume of fluid out ahead of the surfactant, forming a rarefaction wave interacting with a shock, both solutions of (7.2) and decaying in time. For larger values of $h_{R}$, as in Figure 13(c), there is also a leading shock being eroded by the rarefaction wave behind it, once again ahead of the surfactant. 
Height $h$

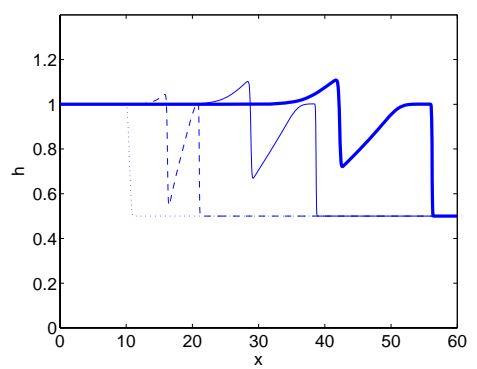

(a) $h_{R}=0.5$

(above the threshold $h_{R}=\frac{\sqrt{3}-1}{2}$ ).

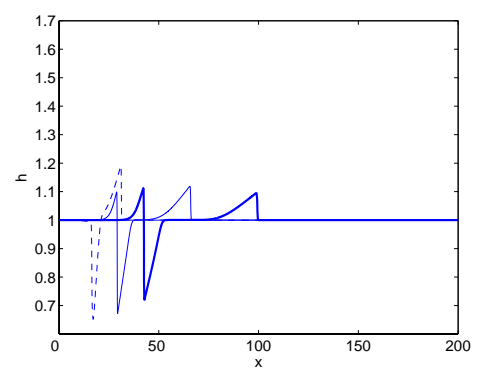

(b) $h_{R}=1.0$

(initially flat film).

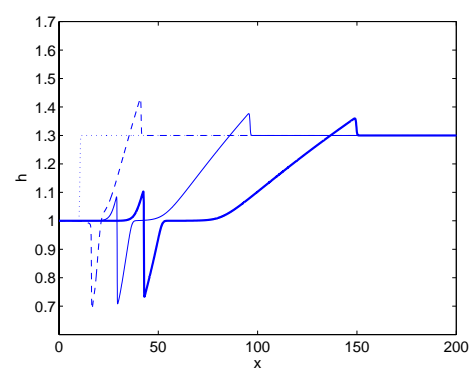

(c) $h_{R}=1.3$.
Surfactant Concentration $\Gamma$

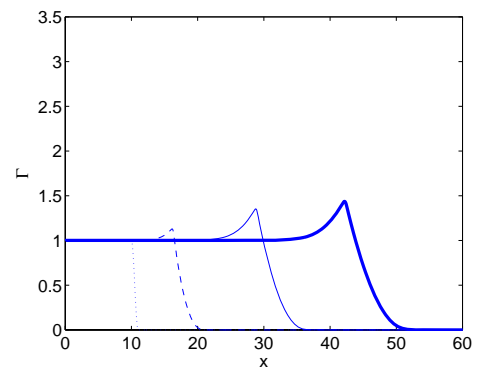

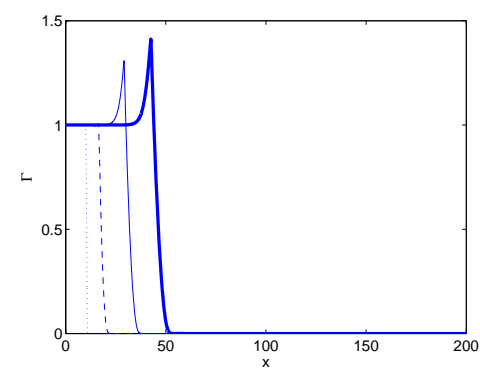

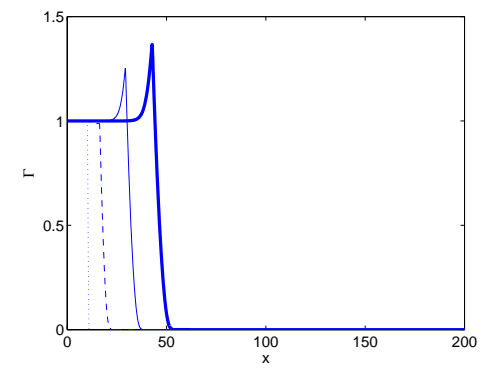

FIG. 13. Variation in wave structure for fixed upstream height $h_{L}=1$ and various downstream heights $h_{R}$. Each plot has graphs for $t=0$ (dotted line), $t=15$ (dashed line), $t=45$ (thin line), and $t=75$ (bold line).

The structure of the surfactant profiles is remarkably unchanged over the entire range of $h_{R}$. However, $\Gamma$ appears to reach $\Gamma=0$ smoothly without the corner observed in simulations with $h_{R}$ below the critical value.

8. Discussion. The mixed hyperbolic-parabolic system of this paper is derived from the lubrication approximation for the influence of surfactant on flow of a thin liquid film on an inclined plane, neglecting smoothing terms of capillarity and surface diffusion. The analysis of traveling waves and jump conditions leads to the identification of a variety of individual waves.

There are traveling waves in which $h$ and $\Gamma$ are smooth and nonlinear (Theo- 
rem 4.2), and there are three cases in which $h$ is piecewise constant and $\Gamma$ is piecewise linear:

Neither $h$ nor $\Gamma_{x}$ jumps (Theorem 4.1). $h$ is constant and $\Gamma$ is linear.

Only h jumps (Theorem 5.6(a)). In numerical simulations, we find that jumps down are stable and jumps up are unstable. The stable waves are counterpropagating in that the thin film and surfactant flow up the inclined plane but the jump propagates downwards.

Both $h$ and $\Gamma_{x}$ jump (Theorems 5.4 and 5.6). Jumps at $\Gamma=0$, the leading edge of the surfactant, are related to the step in film height discovered by Borgas and Grotberg [3] for horizontal substrates.

We have constructed an exact solution of the PDE with three jumps that is piecewise constant in $h$ and piecewise linear in $\Gamma$ and propagates with constant speed. However, this construction is possible only for the ratio of downstream to upstream height below a critical value $r^{*}$. This ratio also limits the construction of wave combinations that mimic numerical simulations of initial value problems in which surfactant is supplied from upstream. The supply of surfactant from the boundary has the effect of allowing the maximum surfactant concentration to grow without bound, as shown in Figure 4. This effect is not explained by the analysis, but Taylor series expansions can be used to capture the increase locally in space and time [14].

Above the critical ratio, solutions approach a different structure that we plan to analyze in a future paper. These solutions have a distinctive $Z$-wave pattern, together with precursor waves propagating into the undisturbed film with no surfactant. As the $Z$-wave pattern forms, the film height has large dips, which might lead to dewetting. This is a concern in surfactant replacement therapy [13], in which a coating of surfactant is required for healthy lung function. This risk of dewetting does not occur for boundary height ratios below the critical ratio.

While we have discussed stability of waves numerically and to some extent analytically, much remains to be done. It would be interesting to analyze stability of the individual waves for $C>0$ and $D>0$. In a future paper, we plan to analyze the constant volume case in which a thin liquid drop on an inclined substrate is spread by the influence of surfactant and gravity.

Acknowledgments. We would like to thank Barry Edmonstone, Richard Craster, and Omar Matar, who introduced us to the problem presented in this paper and who generously shared preliminary versions of their papers. We also thank Andrea Bertozzi and Tom Witelski for helpful suggestions regarding numerics.

\section{REFERENCES}

[1] R. ARIs, Vectors, Tensors, and the Basic Equations of Fluid Dynamics, Prentice-Hall, Englewood Cliffs, NJ, 1962.

[2] A. L. Bertozzi, A. Münch, and M. Shearer, Undercompressive shocks in thin film flows, Phys. D, 134 (1999), pp. 431-464.

[3] M. S. Borgas And J. B. Grotberg, Monolayer flow on a thin film, J. Fluid Mech., 193 (1988), pp. $151-170$.

[4] A. M. Cazabat, F. Heslot, S. M. Troian, and P. Carles, Fingering instability of thin spreading films driven by temperature-gradients, Nature, 346 (1990), pp. 824-826.

[5] A. Dominguez, A. Fernandez, N. Gonzalez, E. Iglesias, and L. Montenegro, Determination of critical micelle concentration of some surfactants by three techniques, J. Chem. Educ., 74 (1996), pp. 1227-1232.

[6] B. D. Edmonstone, R. V. Craster, and O. K. Matar, Flow of surfactant-laden thin films down an inclined plane, J. Engrg. Math., 50 (2004), pp. 141-156. 
[7] B. D. Edmonstone, R. V. Craster, and O. K. Matar, Surfactant-induced fingering phenomena in thin film flow down an inclined plane, Phys. D, 209 (2005), pp. 62-79.

[8] X. Fanton, A. M. Cazabat, And D. Quere, Thickness and shape of films driven by a Marangoni flow, Langmuir, 12 (1996), pp. 5875-5880.

[9] B. J. Fischer And S. M. TRoian, Thinning and disturbance growth in liquid films mobilized by continuous surfactant delivery, Phys. Fluids, 15 (2003), pp. 3837-3845.

[10] D. Halpern, J. L. Bull, And J. B. Grotberg, The effect of airway wall motion on surfactant delivery, J. Biomech. Eng., 126 (2004), pp. 410-419.

[11] D. Halpern, O. E. Jensen, And J. B. Grotberg, A theoretical study of surfactant and liquid delivery into the lung, J. Appl. Physiol., 1 (1998), pp. 333-352.

[12] O. E. Jensen, Self-similar, surfactant-driven flows, Phys. Fluids, 6 (1994), pp. 1084-1094.

[13] O. E. JENSEN AND J. B. GROTBERG, Insoluble surfactant spreading on a thin viscous film: Shock evolution and film rupture, J. Fluid Mech., 240 (1992), pp. 259-288.

[14] R. Levy, Partial Differential Equations of Thin Liquid Films: Analysis and Numerical Simulation, Ph.D. thesis, North Carolina State University, Raleigh, NC, 2005.

[15] R. Levy And M. Shearer, Kinetics and nucleation for driven thin film flow, Phys. D, 209 (2005), pp. 145-163.

[16] O. K. Matar and S. M. Troian, Spreading of a surfactant monolayer on a thin liquid film: Onset and evolution of digitated structures, Chaos, 9 (1999), pp. 141-153.

[17] M. RENARDY, On an equation describing the spreading of surfactants on thin films, Nonlinear Anal., 26 (1996), pp. 1207-1219.

[18] M. Renardy, A singularly perturbed problem related to surfactant spreading on thin films, Nonlinear Anal., 27 (1996), pp. 287-296.

[19] H. Stone, A simple derivation of the time-dependent convective-diffusion equation for surfactant transport along a deforming interface, Phys. Fluids A, 2 (1990), pp. 111-112.

[20] S. M. Troian, E. Herbolzheimer, S. A. Safran, and J. F. Joanny, Fingering instabilities of driven spreading films, Europhys. Lett., 10 (1989), pp. 25-30. 